\title{
Approaches for modeling ionosphere irregularities based on the TEC rate index
}

\author{
Iurii Cherniak ${ }^{1,2^{*}}$, Irina Zakharenkova ${ }^{1,2}$ and Andrzej Krankowski ${ }^{1}$
}

\begin{abstract}
The ionosphere plays an important role in GNSS applications because it influences radio wave propagation. The ionospheric delay is the biggest error source for satellite navigation signals, but it can be directly measured and mitigated using dual-frequency GNSS receivers. However, the GNSS signal fades because of electron density gradients and irregularities in the ionosphere, decreasing the operational performance of navigation systems. Recently, several models were developed to reproduce the ionospheric fluctuations and scintillation activity under different geophysical conditions, but these models were calibrated with data sets without GNSS-derived experimental total electron content (TEC) data. There is a great demand for a proper model of ionospheric irregularity specification based on GNSS TEC measurements. In this work, we use data from the permanent GNSS network to develop the empirical model of the ionospheric irregularities over the Northern Hemisphere. As initial data, we used the daily dependences of the rate of TEC index (ROTI) as a function of geomagnetic local time on the specific grid. The ROTI maps allow us to estimate the overall fluctuation activity and the auroral oval evolution. The irregularities of the southern oval border were determined with the ROTI. This paper presents the correlation between the Kp geomagnetic index and parameters that characterized the activity of the ionosphere irregularities in 2010 to 2013.
\end{abstract}

Keywords: Ionosphere; TEC fluctuations; Irregularities oval; Empirical model; Space weather

\section{Introduction}

The ionospheric fluctuations and irregularities, together with their effects on the GNSS applications, have been studied extensively over the last few decades (Kintner et al. 2007; Beniguel and Adam, 2007; Prikryl et al. 2014), but the current navigation systems are still vulnerable to strong TEC fluctuation events. The ionospheric activity in polar, sub-auroral, and mid-latitude regions is primarily associated with geomagnetic storms, coronal mass ejections, and coronal holes. Processes in the ionosphere during these events result in the formation of horizontal TEC gradients with TEC enhancement, which lead to phase and amplitude scintillation, as reported by many researchers (Vo and Foster, 2001; Stankov et al. 2006; Jakowski et al. 2004; Krankowski et al. 2006; Jakowski et al. 2008; Shagimuratov et al. 2012). Usually, GPS positioning requires a minimum of four simultaneously tracked satellites with good geometry (Misra and

\footnotetext{
* Correspondence: tcherniak@ukr.net

${ }^{1}$ Space Radio-Diagnostic Research Center, University of Warmia and Mazury, Olsztyn, Poland

${ }^{2}$ West Department of Izmiran, Kaliningrad, Russia
}

Enge, 2006). If electron density irregularities cover a large area above a receiver, there is a high probability that a receiver can lose more than one satellite signal simultaneously. Simultaneous loss of a significant number of satellite signals discontinues GNSS navigation or degrades the navigation accuracy. Therefore, strong ionosphere TEC gradients and the resulting fluctuations and signal scintillations can be hazardous in terms of continuity and availability for GNSS navigation (Forte and Radicella, 2004).

During the last few decades, several models were developed to represent the ionospheric fluctuations and scintillation activity under different geophysical conditions. The wide band model (WBMOD) describes a worldwide climate of ionospheric plasma density irregularities (Secan et al. 1995). The parameters of the ionospheric irregularities are modeled on the basis of experimental data. This model provides the intensity scintillation index, S4, and the phase scintillation index, computed by means of the propagation model under pre-specified geophysical conditions. The Global Ionospheric Scintillation Model (GISM) provides the statistical characteristics of the transmitted signals, in particular, the scintillation indices. Maps of the 
modeled scintillation indices can be easily obtained (Beniguel, 2002).

WBMOD and GISM are theoretical models calibrated with data on the global morphology of scintillation activity derived from combining measurement data from the VHF and L band links; these calibration datasets did not include GNSS data. The main limitation of these scintillation models is that they are based on the weak scattering theory and, even when modeling an irregularity on a given link in the best possible way, they are limited to low scintillation values. Their weak scattering approximation works well only when the electron density fluctuations are not very high, about few tens of percent, and cannot represent cases of very high scintillation activity, which are responsible for loss of signal lock and consequent degradation of GPS positioning and navigation operations (Forte and Radicella, 2004).

It is therefore very important to assimilate the GNSSbased fluctuation data into the existing theoretical model by means of new data calibration and to develop a new empirical or semi-empirical model based on GNSS TEC measurements of the ionospheric fluctuations and scintillation.

Ionospheric effects are typically characterized by measuring their impact on the amplitude and phase of the received GNSS signal. The most commonly used measures are the scintillation indices (i.e., amplitude and phase) and the TEC rapid changes (rate of TEC).

The amplitude scintillation index, S4, is the standard deviation of the normalized signal intensity. TEC, defined as the line integral of the electron density from a GPS satellite to a receiver, can be estimated from the differences between the carrier phases of two frequencies (Blewitt, 1990).

To study the amplitude scintillation, we need scintillation receivers that are able to provide direct measurements of the scintillation indices. Currently, there are several extensively developed networks equipped with such receivers, e.g., the Scintillation Network Decision Aid run by the Air Force Research Laboratory (AFRLSCINDA), covering mainly the equatorial region, the invert scintillation receiver network spread in the meridional direction and operated by the University of Bath, the Canadian High-Arctic Ionospheric Network (CHAIN), and a few small regional networks. These networks are rather expensive and are mainly used to evaluate the effects of local ionospheric scintillation on critical military space communication and navigation, and they have specific limitations for data access.

The low-frequency phase fluctuations can be directly estimated by the TEC changes along the ray path for a dual-frequency receiver. The International Geodesy Service (IGS) network provides GNSS observations with $30 \mathrm{~s} \mathrm{sam-}$ pling and allows detection of irregularities with a scale size of about tens of kilometers. These phase fluctuations can be measured using thousands of permanent GNSS stations around the globe. For these purposes, the rate of TEC (ROT) and the rate of TEC index (ROTI) are calculated from RINEX data files using the method proposed by Pi et al. (1997). It is well known that these parameters describe the dynamic changes in the electron density derived from a single-session GNSS satellite receiver. Both ROTI and S4 are important indices in monitoring ionospheric irregularities. ROTI corresponds well with the trend of S4 indices during the generation, evolution, and decay phases of signal amplitude scintillation. Based on the results reported by $\mathrm{Pi}$ et al. (1997), Basu et al. (1999), and Beach and Kintner (1999), it was concluded that ROTI could be used as a proxy for assessing the presence of ionospheric scintillation. Therefore, ROTI can be considered an effective indicator of the occurrence of both phase fluctuations and amplitude scintillation in the high latitude ionosphere. The empirical model of ionospheric irregularities based on ROTI data is actually for space weather studies and transionospheric radio propagation applications, i.e., estimation, specification, and prediction of the probability and area of ionospheric irregularity development and their dependence on solar and geomagnetic activity.

\section{Data and methodology}

According to the recommendation of the IGS Ionosphere Working Group, our group has developed a methodology and service that provide estimation of the ionospheric fluctuation activity based on ROT/ROTI calculations (Cherniak et al. 2014). In order to collect data on TEC fluctuation activity over the North Pole, the Northern Hemisphere polar, sub-auroral, and mid-latitude regions geographically located from $45^{\circ} \mathrm{N}$ geomagnetic latitude to the North Pole were considered. As an initial database for ROTI map calculations, we involved observations from more than 700 permanent stations that are available from IGS, UNAVCO consortium, and European Terrestrial Reference System (EUREF) services (Figure 1a).

Monitoring the time derivative of TEC (ROT) is useful for tracing the occurrence of ionospheric irregularities. ROT, as a measure of phase fluctuation activity, is calculated using the algorithm proposed by Pi et al. (1997). The ROT values are calculated for every considered GNSS station and then detrended for all individual satellite tracks for elevation angles greater than $20^{\circ}$. ROTI, defined as the standard deviation of the detrended ROT values (Pi et al. 1997), can then effectively characterize the ionospheric fluctuation activity. Based on the retrieved values of ROT, the ROTI values are calculated over 5 -min periods with a sliding window.

In order to reveal the spatial structure of the ionospheric irregularities over the North Pole, we process 


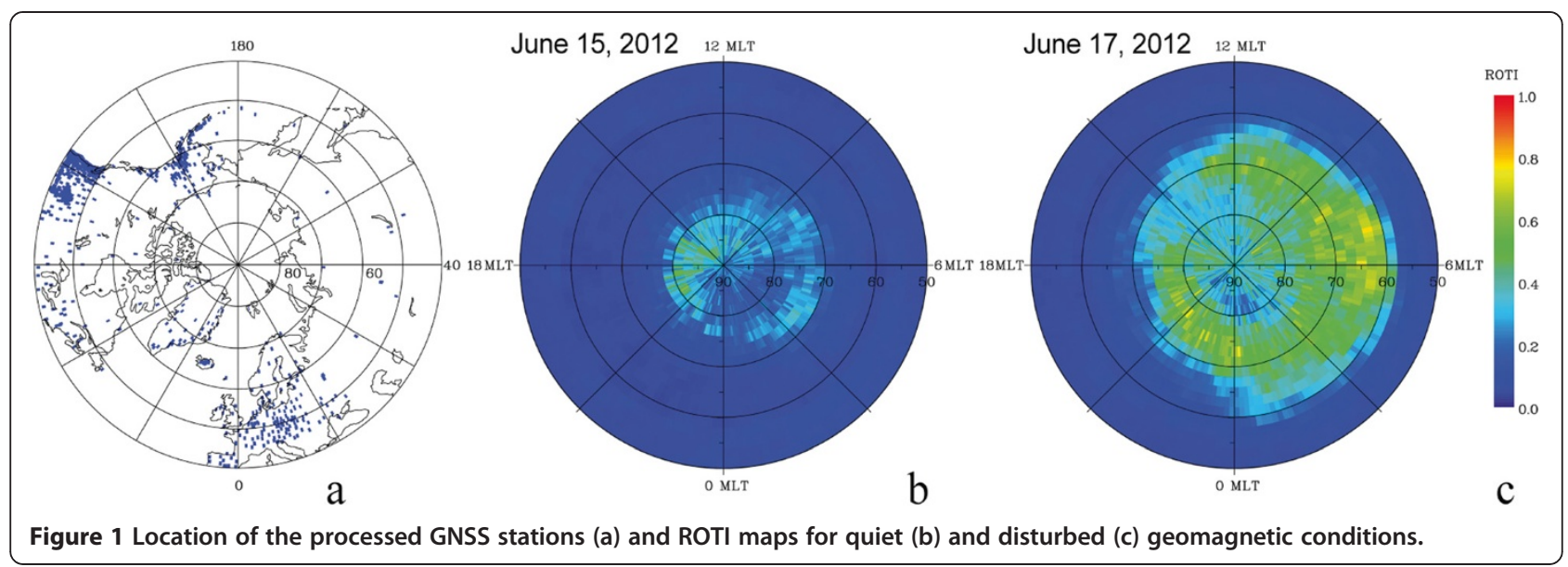

ROTI data from the considered multi-site database and visualize the results in the form of a ROTI map. Because of close interconnection between the Earth's magnetic field and the ionosphere, the behavior of the fluctuation occurrence is represented as a function of the magnetic local time (MLT) and the corrected magnetic latitude. Each map, as a daily map, demonstrates the dynamics of ROTI variation within geomagnetic local time (00-24 MLT). ROTI maps are constructed with a grid of $2^{\circ} \times 2^{\circ}$ resolution. The value in each cell is calculated by averaging over all ROTI values covered by this cell area, and it is proportional to the fluctuation event probability within the current sector.

Figure 1b,c shows a sample ROTI map. These maps correspond to quiet (Figure $1 \mathrm{~b}, \Sigma \mathrm{Kp}=3$ ) and geomagnetically disturbed (Figure 1c, $\Sigma \mathrm{Kp}=37$ ) days in June 2012.

It is evident that during a storm, the intensity of TEC fluctuations is essentially increased. The irregularity oval expands equatorward with increased magnetic activity. The occurrence of irregularities is strongly dependent on geomagnetic activity. Therefore, using the GPS network and the GPS phase fluctuation index ROTI, it is possible to monitor global activity of the ionospheric irregularities. Such maps can be used to find the locations of ionospheric irregularities, as well as the severity of their effects on GPS signals, in the frame of latitude and local time. A similar technique is used by the joint JPL University of Alaska team (Valant-Spaight et al. 2013).

\section{Results and discussions}

Analysis of the occurrence of ionospheric irregularities was based on daily ROTI maps for the period 2010 to 2013, which included the peak of the 24th solar cycle. However, this period is characterized by moderate geomagnetic activity. The results reveal that the main features of the irregularity pattern are the ROTI index intensity and the position of the irregularity oval's southern border (Cherniak et al. 2014). These parameters depend strongly on space weather conditions, such as solar wind parameters and geomagnetic indices. To estimate the day-to-day fluctuation activity dependences (and diurnal ROTI maps) from the geomagnetic conditions, it is convenient to use the sum of $\mathrm{Kp}$ index values over the day considered.

As a measure of the overall fluctuation activity for the selected region, we use the Hemisphere ROTI index (HROTI, daily values) that takes into account all fluctuation events from mid-latitude to auroral regions. The idea of the HROTI index for ionospheric irregularities is similar to the global electron content (GEC) index proposed by Afraimovich et al. (2008), where GEC is calculated by summation of the TEC values multiplied by the cell's area. The HROTI is calculated according to the equation:

$$
\text { HROTI }=\Sigma \text { ROTI }_{\mathrm{ij}} \bullet \mathrm{S}_{\mathrm{ij}}
$$

where $\mathrm{ROTI}_{\mathrm{ij}}$ is the ROTI index in the current cell and $\mathrm{S}_{\mathrm{ij}}$ is the cell size.

Figure 2 presents the correlation between $\mathrm{HROTI}_{\mathrm{ij}}$ and the daily sum of Kp geomagnetic indices $(\Sigma K \mathrm{~K})$. The strong dependence between the fluctuation activity index and the Kp index is clearly seen. There is a strong correlation $(\mathrm{R}=0.79)$ between $\Sigma \mathrm{Kp}$ and HROTI, and HROTI values can be modeled using a linear predictor function (linear regression model). The linear regression equation with the corresponding coefficients is presented in Figure 2.

In order to specify the position of the irregularities at the oval's southern (equatorward) border, we developed algorithms for determining the shape and position of the southern border of the oval. These algorithms are based on analysis of the ROTI values over the whole ROTI map along a selected set of cells from south to north.

The dependences of the position of the southern border of the ionospheric irregularities oval (SBIR) were 


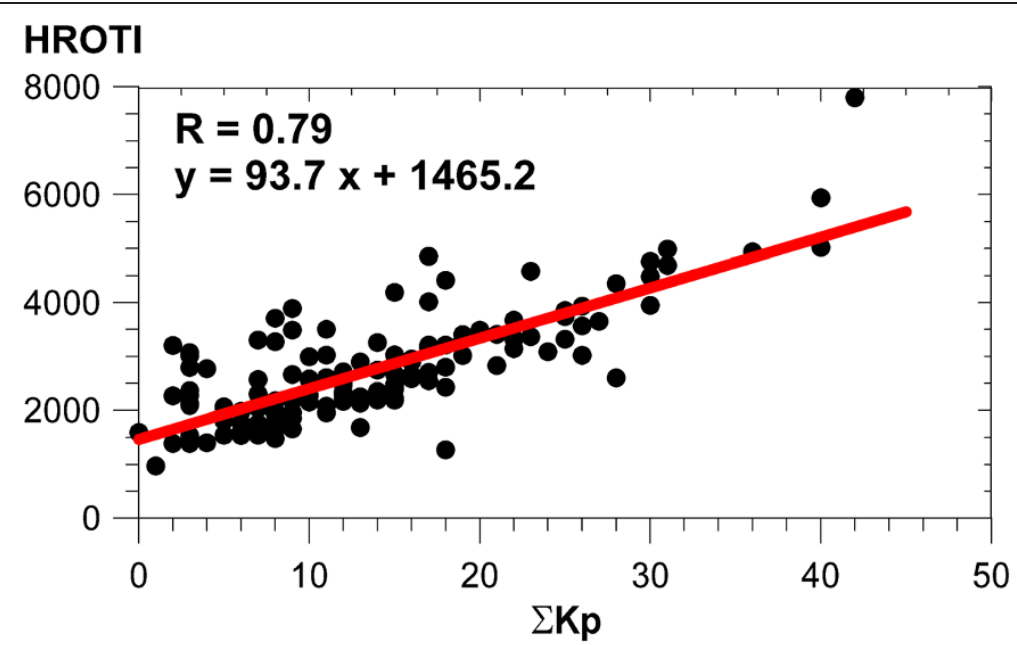

Figure 2 Scatter plot of HROTI index with sum Kp; $\mathbf{R}$ is the correlation coefficient. The red line corresponds to the best fit line.

analyzed for the period 2010 to 2013. Figure 3 presents the SBIR position for different values of the daily sum of geomagnetic indices $\mathrm{Kp}$. We examined several intervals with different sums of Kp values to construct the SBIR positions (indicated by red line). The solid black lines indicate the standard deviations of the calculated values.

These graphs illustrate that for non-disturbed conditions $(\Sigma K \mathrm{p}<10)$, all irregularities were located in the night sector and near the cusp region; the oval border did not cross 70 MLAT and was extended to the morning sector. The averaged HROTI index for such conditions did not exceed the value of 2,000. When the sum of Kp values was within 10 to 20 limits, the SBIR expanded up to 75 MLAT in the night sector. With the increase of geomagnetic activity, the fluctuation activity also increased (the index value was more than 4,000 for
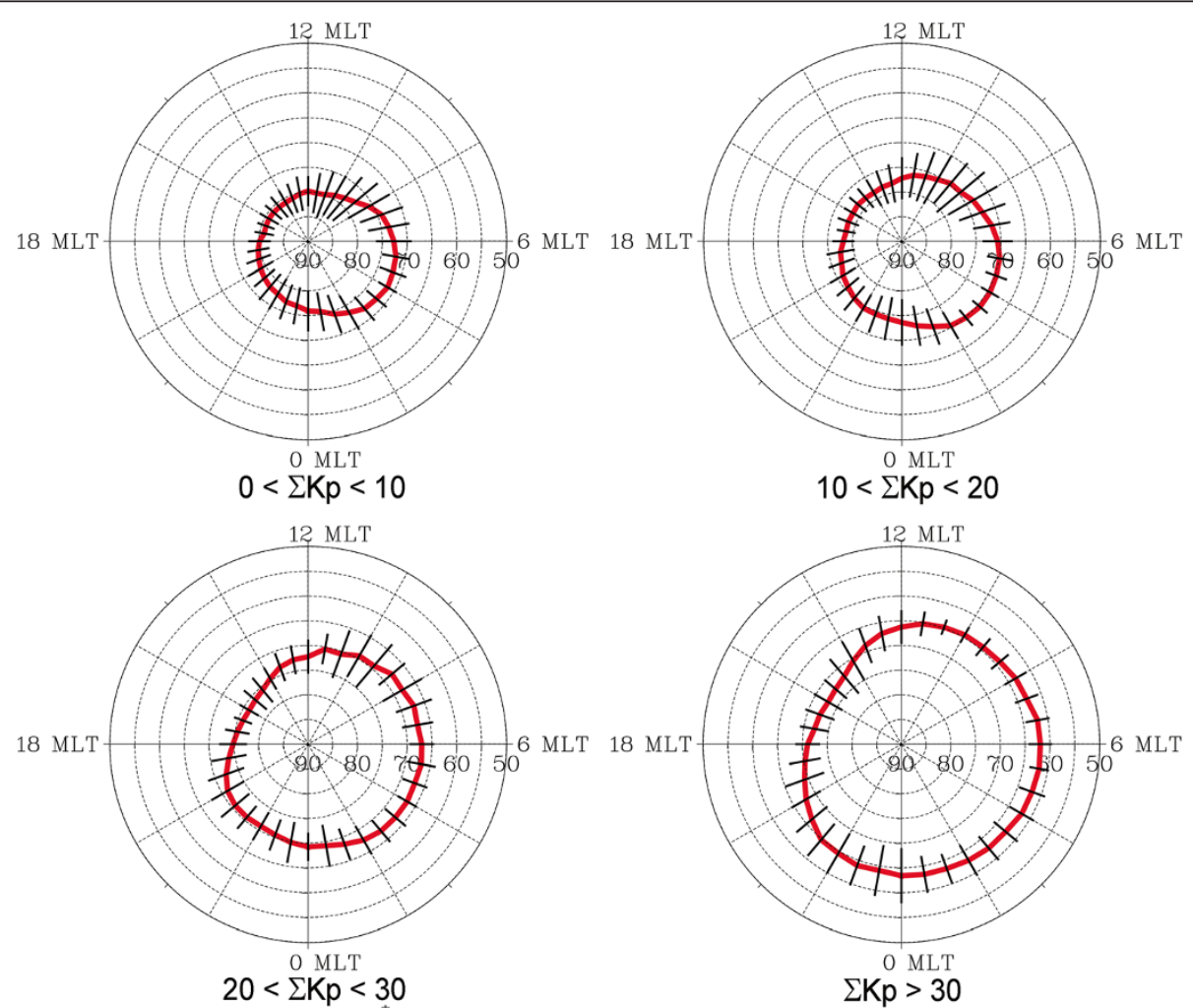

Figure 3 Southern border of the ionospheric irregularities oval for different levels of geomagnetic activity. 
$\Sigma \mathrm{Kp}>30$ ), and the SBIR expanded up to $65^{\circ}$ to $60^{\circ}$ MLAT, and could cover sectors from 23 to 8 MLT. For disturbed geomagnetic conditions when $\Sigma \mathrm{Kp}>20$, the SBIR can extend equatorward to $75^{\circ}$ to $70^{\circ}$ MLAT, even in the daytime sector.

The obtained statistical dependences, correlation coefficients, and regression equations are used as the initial part of the ionospheric fluctuation model being developed by our team.

\section{Summary}

Analysis and specification of ionospheric irregularities at mid- and high latitudes using GNSS measurements represent an important task for both scientific and GNSS applications, as the occurrence of ionospheric irregularities can impact the performance of communication and navigation systems. Thus, knowledge of the morphology and dynamics of ionospheric irregularities, as well as their dependence on geophysical factors, is very important for mitigating space weather effects on GNSS.

The approaches considered here are based on mapping ionospheric irregularities and can effectively solve the task of fluctuation activity estimation, as well as the levels and locations of large-scale irregular structures. The indices and maps, derived using TEC rate changes, can be used as sensible and very sensitive indicators of the occurrence of phase fluctuations is the high and mid-latitude ionosphere.

The results of our study demonstrate that it is possible to use the current network of GNSS permanent stations to estimate the intensity of ionospheric irregularities, which can be described by ROTI (corresponding ROTI maps and HROTI), and the position of the irregularity oval's southern border. In our study, the correlation dependences and linear regression coefficients between these parameters and the geomagnetic index Kp (daily sum of $\mathrm{Kp}$ ) were also obtained.

These results can be applied to retrospective estimation of the impact of space weather on GNSS positioning and for development of new ionospheric irregularity models.

\section{Competing interests}

The authors declare that they have no competing interests.

\section{Authors' contributions}

IC designed the study, developed the methodology, performed the analysis, and drafted the manuscript. IZ developed algorithms and software for data processing and statistical analysis. AK collected and processed initial data. All authors read and approved the final manuscript.

\section{Acknowledgements}

The authors thank IGS, UNAVCO, and EUREF for making available the GPS measurement data and the World Data Center for Geomagnetism, Kyoto for geomagnetic data service.

Received: 30 April 2014 Accepted: 30 November 2014

Published online: 12 December 2014

\section{References}

Afraimovich EL, Astafyeva El, Oinats AV, Yasukevich YV, Zhivetiev IV (2008) Global electron content: a new conception to track solar activity. Ann Geophys 26(2):335-344

Basu S, Groves KM, Quinn JM, Doherty P (1999) A comparison of TEC fluctuation and scintillations at Ascension Island. J Atmos Sol Terr Phys 61:1219-1226

Beach TL, Kintner PM (1999) Simultaneous Global Position System observations of equatorial scintillations and total electron content fluctuations. J Geophys Res 104(A10):22553-22565

Beniguel Y (2002) GISM: a propagation model for scintillations of transmitted signals. Radio Sci 37(3), doi:10.129/2000RS002393

Beniguel Y, Adam J-P (2007) Effects of scintillations in GNSS operation. Astrophysics and Space Science Library, vol 344. (e-book), Springer, Netherlands, doi:10.1007/ 1-4020-5446-718. ISBN 978-1-4020-5445-7

Blewitt G (1990) An automatic editing algorithm for GPS data. Geophys Res Lett 17:199-202

Cherniak I, Krankowski A, Zakharenkova I (2014) Observation of the ionospheric irregularities over the Northern Hemisphere: methodology and service. Radio Sci, doi:10.1002/2014RS005433

Forte B, Radicella S (2004) Geometrical control of scintillation indices: what happens for GPS satellites. Radio Sci 39(RS5014), doi:10.1029/2002RS002852

Jakowski N, Leitinger R, Ciraolo L (2004) Behavior of large scale structures of the electron content as a key parameter for range errors in GNSS applications. Ann Geophys 47(2/3):1031

Jakowski N, Mielich J, Borries C, Cander L, Krankowski A, Nava B, Stankov SM (2008) Large scale ionospheric gradients over Europe observed in October 2003. J Atm Sol Terr Phys 70(15):1894-1903

Kintner, P.M., Ledvina, B.M. and de Paula, E.R. (2007) GPS and ionospheric scintillations. Space Weather 5. doi:10.1029/2006SW000260

Krankowski A, Shagimuratov I, Baran L, Efishov I, Tepenitzyna N (2006) The occurrence of polar cap patches in TEC fluctuations detected using GPS measurements in Southern Hemisphere. Adv Space Res 38:2601-2609

Misra P, Enge P (2006) Global Positioning System: signals, measurements, and performance. Ganga-Jamuna Press, Lincoln, MA

Pi X, Mannucci AJ, Lindqwister UJ, Ho CM (1997) Monitoring of global ionospheric irregularities using the worldwide GPS network. Geophys Res Lett 24:2283

Prikryl P, Jayachandran T, Mushini S, Richardson I (2014) High-latitude GPS phase scintillation and cycle slips during high-speed solar wind streams and interplanetary coronal mass ejections: a superposed epoch analysis. Earth, Planets Space 66:62, doi:10.1186/1880-5981-66-62

Secan JA, Bussey RM, Fremouw EJ, Basu SA (1995) An improved model of equatorial scintillation. Radio Sci 30:607-617

Shagimuratov II, Krankowski A, Ephishov I, Cherniak I, Wielgosz P, Zakharenkova I (2012) High latitude TEC fluctuations and irregularity oval during geomagnetic storms. Earth, Planets Space 64(6):521-529

Stankov SM, Jakowski N, Tsybulya K, Wilken V (2006) Monitoring the generation and propagation of ionospheric disturbances and effects on GNSS positioning. Radio Sci 41:RS6S09

Valant-Spaight B, Pi X, Mannucci A, Martin Hal G (2013) Monitoring ionospheric scintillation effects on precise positioning in the North America region. Space Weather Workshop, USA, Colorado, Boulder

Vo HB, Foster JC (2001) A quantitative study of ionospheric density gradients at midlatitudes. J Geophys Res 106(A10):21555-21563

doi:10.1186/s40623-014-0165-z

Cite this article as: Cherniak et al:: Approaches for modeling ionosphere

irregularities based on the TEC rate index. Earth, Planets and Space 2014 66:165. 